\title{
HILARY PUTNAM, LA EVOLUCIÓN DEL REALISMO Y LA PERSPECTIVA PRAGMATISTA
}

\author{
HiLARY PUTNAM, THE EVOLUTION OF REALISM AND THE \\ PRAGMATIST POINT OF VIEW
}

Juan V. Mayoral

\begin{abstract}
RESUMEN
Este ensayo es una introducción al número de la revista Análisis dedicado a la filosofía de Hilary Putnam. En él se aporta una introducción temática a la filosofía de este autor, con breves matices acerca de su desarrollo intelectual. El ensayo comienza por su trabajo con el realismo, ya sea metafísico, interno o natural; continúa exponiendo las relaciones de Putnam con los autores del pragmatismo, en especial con William James y John Dewey; y, para finalizar, muestra la postura de Putnam sobre la interpretación de la teoría cuántica. En cada sección se exponen brevemente los contenidos de los ensayos que conforman este número y su relación con dichas divisiones temáticas.
\end{abstract}

PALABRAS CLAVE: Putnam, Dewey, James, realismo metafísico, realismo interno, realismo natural, a priori, relatividad conceptual, pragmatismo, teoría cuántica.

\begin{abstract}
This essay is an introduction to the issue of the journal Análisis devoted to the philosophy of Hilary Putnam. This paper provides a thematic introduction to Putnam's thought, though some indications concerning his intellectual development are also included. It begins with Putnam's work on realism, whether metaphysical, internal or natural; then it deals with Putnam's relations with classic authors of American pragmatism as William James and John Dewey. In the last section, before concluding remarks, Putnam's position concerning the interpretation of quantum mechanics is briefly shown. Each section includes an exposition of the essays that make up this issue as well as their relations with the themes previously expounded.
\end{abstract}

KEYWORDS: Putnam, Dewey, James, metaphysical realism, internal realism, natural realism, a priori, conceptual relativity, pragmatism, quantum theory. 


\section{INTRODUCCIÓN}

Hilary Putnam, uno de los filósofos norteamericanos más prolíficos del último siglo, fallecía hace ahora un poco más de dos años, el 13 de marzo de 2016, en Arlington, en el estado de Massachusetts. Putnam había estudiado alemán, filosofía y análisis lingüístico en la Universidad de Pensilvania en los años cuarenta hasta obtener su título de graduado en 1948. Pasó brevemente por la Universidad de Harvard, donde conoció a W. V. Quine, y se doctoró en 1951 en la Universidad de California, Los Ángeles (UCLA), bajo la dirección del filósofo lógico-empirista Hans Reichenbach. Tras su contratación, ya como profesor, en otras universidades como la de Princeton o el Instituto Tecnológico de Massachusetts (MIT), volvió a Harvard donde pasó la mayor parte de su trayectoria profesional. ${ }^{1}$

En los 65 años que dedicó a la filosofía, Putnam dejó su impronta en terrenos que van de la filosofía de la mente a la filosofía de la ciencia y sin olvidar, por supuesto, sus cruciales contribuciones a la filosofía del lenguaje (en especial, a la teoría de la referencia). ${ }^{2}$ A duras penas podemos hallar un campo de interés en la filosofía analítica del siglo XX en el que Putnam no ofreciese sus opiniones filosóficas. Pero lo mismo podemos decir de otros ámbitos de la filosofía que no se someten con la misma docilidad al marco del análisis conceptual, como, por ejemplo, el estudio de los pragmatistas clásicos, de Charles S. Peirce y William James a John Dewey. ${ }^{3}$

Dada esa amplitud de intereses, en este número de Análisis que dedicamos a su obra, para el que este ensayo sirve de presentación, solo nos es posible tratar con cierto detalle un número limitado de cuestiones de entre todas las tratadas por Putnam a lo largo de su vida. Con todo, las que aquí se han seleccionado son representativas de aspectos y periodos centrales de su pensamiento y especialmente apropiadas para comprender su desarrollo intelectual y los cambios en los enfoques filosóficos por él adoptados y defendidos. En los ensayos que siguen, los tres temas fundamentales se agrupan cómodamente bajo las denominaciones

\footnotetext{
1 Muchos de los datos biográficos empleados en esta primera sección introductoria, empezando por los que acabo de emplear, se basan en Baghramian (2013a, 2016) y en Putnam (2012a, 2013a, 2013b y 2015).

2 Sobre esta última, son bien conocidos algunos de los artículos clave que reunió más adelante en el segundo volumen de sus Philosophical Papers. Sobre su trayectoria en torno a la última materia citada, la filosofía del lenguaje, of. Putnam (2016a).

3 Véanse, a este respecto, los artículos compilados recientemente en Pragmatism as a Way of Life, escritos por él y por su esposa, Ruth Anna Putnam (Putnam y Putnam 2017).
} 
temáticas incluidas en el título de este número: el realismo, el pragmatismo y la teoría cuántica. La intención de este primer ensayo de presentación es exponer un contexto temático que integre los estudios especializados que vendrán a continuación y que muestre que, gracias a ellos, obtenemos una imagen más perfilada de algunos de los aspectos claves de la filosofía de Hilary Putnam.

\section{LAS FORMAS DEL REALISMO}

La postura realista implica, en su aspecto más básico, que nuestras creencias, o las proposiciones de nuestro lenguaje, son verdaderas (o falsas) debido a una relación que se da (o no, si son falsas) con el mundo "externo", esto es aquel que es ajeno o bien al lenguaje, o bien a la mente (contemplada esta como algo distinto del mundo), o bien a ambos, y que en un vocabulario kantiano es el substrato nouménico de nuestra experiencia.

Más allá de ello hay otros matices, algunos de los cuales refiere el autor al que dedicamos varios artículos de este número de la revista Análisis: Hilary Putnam. En "A Defense of Internal Realism" (1982), por ejemplo, Putnam considera que el realista metafísico es alguien que cree que: (1) "el mundo se compone de una totalidad fija de objetos independientes de la mente"; que (2) "hay exactamente una descripción verdadera y completa del modo en que es el mundo"; y que (3) "la verdad implica alguna clase de correspondencia" del lenguaje con el mundo." Desde luego, en las fechas en que Putnam mostró esta versión del realismo metafísico, su actitud hacia este era fundamentalmente crítica y ya había optado por defender una postura que para muchos representa exactamente la opción opuesta: el realismo interno, que es más bien una forma de antirrealismo. Pero el punto de partida establecido para su crítica queda así bien fijado y sirve para entender sobre qué esquema inicial trabaja Putnam en los años venideros.

La actitud del realista, en cualquier caso, no se resuelve exclusivamente en los términos que acabamos de exponer, esto es, en un realismo metafísico o en un realismo interno. Sin embargo, todos comparten una misma superación del agnosticismo hacia el mundo externo y nuestra relación con él. Así, por ejemplo, el realismo científico considera que nuestras teorías de mayor éxito en la ciencia,

\footnotetext{
4 Putnam (1982, p. 30). Cf. Putnam (1981, p. 49), para una versión muy semejante. El artículo de Howard Sankey para este número de Análisis ("Putnam's Internal Realism in Retrospect") también se ocupa de esta versión de Putnam y examina en detalle esa distribución tripartita (esp. apdo. 2). Más adelante volveremos sobre esas tesis.
} 
aquellas que hoy usamos y atesoramos como correctas, son, de hecho, verdaderas; es decir, nuestros términos, ya sean observacionales y de sentido común ("mesa" o "gato", por ejemplo), ya sean puramente teóricos (como "electrón"), refieren objetos realmente existentes en el mundo; objetos que "están ahî" incluso aunque no hubiese un observador para acceder a ellos y nombrarlos. Esta es una de las posturas defendidas por Putnam a lo largo de su carrera, desde que expusiese el llamado "argumento del milagro" a mediados de los años 70 del pasado siglo, a saber, aquel que dice que "el realismo es la única filosofía que no hace del éxito de la ciencia un milagro". ${ }^{5}$ El realismo científico explica que la ciencia, tal como hoy la conocemos, presente los rasgos evidentes de eficacia y progreso histórico en el control de la naturaleza que ofrece. ${ }^{6}$ Frente a este realismo científico, podríamos hallar un realismo de sentido común como el que menciona Mario de Caro (2016) y que, en sus palabras, es "la perspectiva que reconoce como real solo las cosas que podemos experimentar directamente". La diferencia entre ambos se basa, principalmente, en la serie de entidades ajenas al lenguaje o la mente con las que el teórico en cuestión está dispuesto a comprometerse. Aunque su oposición puede llegar a ser de mutua exclusión, el proyecto putnamiano (sobre todo en fechas recientes), dice De Caro, es que ambos sean netamente compatibles. ${ }^{8}$

La diversidad de realismos aumenta a medida que somos más precisos a la hora de contemplar qué tesis principales los caracterizan. ${ }^{9}$ Este pequeño mapa es, no obstante, suficiente para mostrarnos entre qué posiciones ha evolucionado Putnam desde sus inicios. La periodización de su pensamiento en torno a la cuestión del realismo (una de las centrales en los artículos que vienen a continuación)

5 Putnam (1975a, p. 73). Cf. Diéguez (1998, pp. 104-105) para una útil explicación del "argumento del milagro" y su cap. 6 para la crítica del realismo metafísico desde el punto de vista del realismo interno, así como el texto de De Caro (2016, p. 8).

${ }^{6}$ Naturalmente, hay tesis en contra de esta postura pro realista, como la tesis de la infradeterminación de las teorías por la evidencia empírica, o el escepticismo metainductivo o la inconmensurabilidad interteórica, en las cuales no entraremos aquí. Para más detalles sobre estas tesis y su interacción con los argumentos a favor del realismo, cf. Diéguez (1998, cap. 5), Psillos (1999) y Sankey (2008c).

7 De Caro (2016, p. 4). De Caro opone este realismo de sentido común a lo que denomina "realismo hipercientífico", que defiende que son "reales solamente las entidades y acontecimientos (observables y no observables) a las que nuestras mejores teorías científicas están comprometidas ontológicamente y ninguna de estas tiene las propiedades que se supone que tienen nuestros objetos de sentido común” (De Caro 2016, pp. 4-5).

${ }^{8}$ Cf. De Caro (2016, pp. 5-8).

9 Cf. Sankey (2008a) y De Caro (2016, p. 4), para más detalles sobre dicha variedad. 
es más o menos aceptada del modo siguiente: el primer periodo va desde sus primeros escritos hacia el comienzo de los años 60 hasta su conferencia "Realism and Reason", de 1976; el segundo va desde esa última fecha hasta principios de los 90 y su final se hace sobre todo manifiesto en sus Conferencias John Dewey de 1994 en la Universidad de Columbia; ${ }^{10}$ el tercero comienza en estos inicios de los 90. Las posturas defendidas en estas fases son, respectivamente, el realismo metafísico, el realismo interno y el realismo natural. ${ }^{11} \mathrm{~A}$ continuación veremos qué matices introduce Putnam acerca de esta periodización.

\section{PUTNAM Y EL REALISMO INTERNO}

El primero de los textos que se muestran a continuación, de Howard Sankey, se ocupa de la postura de Putnam en su segunda fase, esto es, del realismo interno. Antes de pasar a lo que dice Sankey, conviene recuperar un matiz reciente de Putnam sobre el tipo de crítica que supone su propia defensa del realismo interno.

Para Putnam, ha habido cierta confusión en torno a las relaciones de su realismo metafísico, temporalmente defendido por él, con su realismo científico, permanente en su pensamiento. ${ }^{12}$ Putnam considera que el realismo metafísico es, para él, algo muy parecido a un "modelo", que sirve para "la relación de cualquier teoría correcta con todo EL MUNDO o con una parte de él". ${ }^{13}$ Frente a ello, su postura siempre se ha caracterizado por una "teoría empírica" que, en su perspectiva, es lo que el realismo científico es en realidad. ${ }^{14}$ Para Putnam, si ha habido una fase de su pensamiento, la intermedia, en la que el realismo interno se oponía al realismo metafísico, ello no implica que también se opusiese al realismo científico. En su segunda fase, Putnam alteró su comprensión de la verdad, que dejó de ajustarse al canon de la teoría de la correspondencia para equipararse a una "verificación en condiciones ideales" (o "aceptabilidad racional idealizada"; cf. Putnam 2012a, p. 20). Eso supuso un rechazo de una de las tres bases, antes vistas, del canon del realismo metafísico, pero no la alteración de una posición previa, pues, pese a lo

\footnotetext{
${ }^{10}$ Putnam (2015b, p. 485, n. 3) afirma que el final del periodo del realismo interno fue en 1990; en concreto, lo marcó su respuesta a Simon Blackburn en el congreso celebrado en Escocia (Universidad de St. Andrews) ese año y que apareció en Putnam (1994).

11 Sobre esta periodización del pensamiento de Putnam, of. Putnam (1997, 2013b) y Baghramian (2013a, 2016).

12 Sobre la confusión que describimos a continuación, en este párrafo, cf. Putnam (2012a, pp. 20-23).

13 Putnam (1978, p. 123; 1991, p. 142 [v.c.]).

14 Cf. Putnam (1976a, p. 123; 1978, Conf. II).
} 
afirmado en repetidas ocasiones, Putnam avisa de que solo una vez sostuvo explícitamente el realismo metafísico, mientras que, en sus palabras, "siempre me he considerado un realista científico". ${ }^{15}$

El realismo científico es, en efecto, la perspectiva adoptada por Putnam ya a partir de 1960 y, tal como refleja quince años después, en su primer volumen de Philosophical Papers. ${ }^{16}$ No se trataba solo (ni principalmente), matizaba ahí, de defender el realismo científico a capa y espada, sino de emplear ese enfoque con otros objetivos muy diversos. ${ }^{17} \mathrm{Y}$ aunque sus principales temas de investigación son la filosofía de la ciencia y la lógica y la matemática y sus aspectos filosóficos, la ética también había formado parte de sus intereses desde, más o menos, los mismos años en que mostraba abiertamente sus credenciales realistas. Y eso aunque, como él señala, el primero de sus textos que trataba cuestiones éticas fuese Reason, Truth and History (1981). ${ }^{18}$

Putnam cifra su postura a favor del realismo científico en un abandono de las posturas positivistas. ${ }^{19}$ Putnam no solo muestra esa actitud crítica hacia el positivismo en la filosofía de la ciencia, sino también en el terreno de la ética, donde considera (frente a las posturas más positivistas) que las proposiciones de naturaleza ética son un objeto de conocimiento que, no obstante, no se somete a la simplificación, sin duda excesiva, de que el conocimiento genuino es solo conocimiento científico. Aunque su postura en filosofía de la ciencia y de las matemáticas sea inequívocamente realista, Putnam no reduce todo el conocimiento humano a la ciencia, aunque el filósofo debe ocuparse de explorar las muy diversas relaciones entre ambos campos. ${ }^{20} \mathrm{~A}$ medida que Putnam avance en su desarrollo filosófico, su oposición a esos y otros lugares comunes del positivismo lógico (y un buen ejemplo al respecto es la dicotomía hecho/valor, aunque no es el único) se hará aún más manifiesta. Más tarde volveremos sobre ello.

15 Putnam (2012a, p. 22). Cf. De Caro y Macarthur (2012, pp. 22-23), quienes también exponen estos matices.

16 Para Maria Baghramian (2012a, pp. 2-3 y 6), su alejamiento del pensamiento lógico-empirista y de las filosofías de sus dos mentores, Rudolf Carnap y Hans Reichenbach, profundamente influyentes en él durante los años 50, queda establecida en el cambio de década y gracias a sus escritos a partir de 1960.

17 Cf. Putnam (1975b, p. vii).

18 Cf. Putnam (2012a, p. 19).

19 Cf. Putnam (1997, p. 19).

20 Una buena declaración al respecto aparece en Putnam (2013b, p. 215). Sobre lo dicho en el último párrafo, of. Putnam (1975b, pp. xiii-xiv). 
Pero en el periodo intermedio, el periodo del "realismo interno", introduce abruptos cambios en su modo de entender las convicciones realistas. El artículo de Sankey publicado a continuación, "Putnam's Internal Realism in Retrospect", se ocupa de hacer explícitas las bases de esta postura y de ofrecer una crítica y una alternativa. La principal línea de crítica tiene que ver con que el realismo metafísico suponga una postura que represente adecuadamente las convicciones realistas y con que el realismo interno de Putnam sea, de ese modo, una posición alternativa apropiada y, lo que es más importante, afín a tales convicciones.

Para Sankey, de las tres tesis expuestas por Putnam como principios del realismo metafísico (ya mostradas en la segunda sección de nuestro artículo), la número (1) es ya dudosa. Esta hablaba de "una totalidad fija de objetos", pero los discursos que consideramos verdaderos de la ciencia y del sentido común no contemplan el mismo colectivo de objetos y propiedades, ni estos son siempre plenamente compatibles. ${ }^{21}$ Por resumirlo de la siguiente forma: los diversos discursos que la ciencia ofrece en este punto no revelan la idea de "una totalidad fija de objetos"; y, lo que es más importante, de acuerdo con Sankey (y también con Ian Hacking), ni siquiera tienen por qué hacerlo. Como apunta Hacking (1983, p. 95), es posible contemplar que esos diversos discursos de la ciencia y del sentido común ofrecen otras tantas clasificaciones, a veces incompatibles ("inconmensurables", deberíamos decir, con Hacking), de objetos en el mundo, sin que de ello se siga (por usar de nuevo las palabras de Hacking) que "todas esas categorías sean dependientes de la mente". ${ }^{22}$

También hallamos críticas a las tesis (2) y (3) por parte de Sankey. Acabamos de afirmar que nuestros discursos son diversos, esto es, que la ciencia y el sentido común ${ }^{23}$ ofrecen una pluralidad de modos, tal vez incompatibles entre sí, de clasificar el mundo, lo cual contradice el punto (2) visto al principio: que, para la supuesta totalidad de objetos hay "una descripción verdadera y completa". ${ }^{24} \mathrm{Y}$, si finalmente nos referimos a la tesis (3), sobre la teoría correspondencionalista de la verdad, Sankey considera que, si hay un compromiso específico del realista con una teoría

\footnotetext{
21 En este punto, Sankey se apoya en la obra de Ian Hacking (1983, cap. 7, esp. pp. 93-95).

22 Hacking (1985, p. 95; 1996, p. 118 [v.c.]).

${ }^{23} \mathrm{Y}$ ya sabemos que ambos tienen cabida en nuestra perspectiva del conocimiento humano de acuerdo con Putnam.

${ }^{24}$ Para una visión más completa de estas críticas, of. el artículo de Sankey a continuación, así como Sankey (2008b, esp. p. 33).
} 
de la verdad, este es opcional, por lo que podemos contemplar una formulación menos comprometida y más general y esta no tendría en cuenta este aspecto. ${ }^{25}$

Una de las claves del artículo de Sankey se halla en la distribución de lo que es esencial y de lo que es opcional para adoptar una perspectiva realista. Si, como vimos más arriba, Putnam mostraba su afinidad hacia el realismo científico de manera permanente, pero no hacia el realismo metafísico excepto fugazmente a lo largo de toda su obra, la perspectiva de Sankey se aproximaría más a sus fidelidades permanentes. Sankey defiende la utilidad de una visión menos comprometida con tesis de contenido muy restrictivo como las que presentaba Putnam para el realismo metafísico. Esa visión menos comprometida es la de Michael Devitt en Realism and Truth, para quien el realismo es, en esencia, una postura metafísica, no semántica, caracterizada por una afirmación como la siguiente: "los casos de la mayoría de tipos de sentido común y científicos existen” (Devitt 1991, p. 21). En su introducción al primer volumen de los Philosophical Papers (Putnam 1975c, pp. vii-viii), Putnam se compromete con una tesis acerca de la existencia de tales entidades de una manera muy semejante. Su concepción del realismo metafísico va mucho más lejos, sin embargo, a un punto al que Sankey, Devitt o Hacking no están dispuestos a llegar.

La crítica de Sankey consiste, pues, en mostrar que el realismo metafísico de Putnam no constituye una postura extendida en las filas del realismo y, por lo tanto, que el realismo interno es, en todo caso, una propuesta que no actúa frente a los defensores del realismo científico o de sentido común. A decir verdad, vista la defensa del realismo científico y de sentido común por parte de Putnam, como

25 En su "Scientific Realism" (Sankey 2008a), Sankey ofrece una lista de 6 "doctrinas centrales" del realismo científico, entre las que se cuentan (i) el "realismo de objetivos", (ii) el "realismo epistémico", (iii) el "realismo del discurso teórico", (iv) el "realismo metafísico", (v) la "teoría de la verdad como correspondencia” y, por último, (vi) la "objetividad de la verdad" (Sankey 2008a, pp. 13-17). No definiremos aquí cada una de esas doctrinas, pero puede llamar la atención que las (iv) y (v) reaparezcan en Putnam, como ya vimos, y con (v) como parte integrante de (iv). Sankey, no obstante, aclara que su referencia al realismo metafísico no es a una doctrina con las tesis específicas antes vistas, esto es, (1) y (2). Para Sankey, el realismo metafísico solo consiste en que "el mundo investigado por la ciencia es una realidad objetiva que existe independientemente del pensamiento humano" (Sankey 2008a, p. 15). Lo mismo ocurre con (v), la teoría de la verdad basada en la correspondencia, que para Sankey es más bien un marco que incluye tanto la clásica idea de correspondencia como la posibilidad de teorías minimalistas al modo de la de Paul Horwich (1990). Así, para aquel, la teoría de (v) dice que "la verdad consiste en la correspondencia entre una afirmación sobre el mundo y el modo en que es el mundo" (Sankey 2008a, p. 16). 
ya hemos visto, podría parecer que las posturas de Sankey y de Putnam podrían aproximarse. Pero esto último no es posible, al menos en lo que se refiere al periodo intermedio del pensamiento de Putnam, en la medida en que, como muestra Sankey, la identificación de la verdad con la "verificabilidad en condiciones ideales", lo que convierte el primer concepto en una concepción epistémica de la verdad, no es del agrado de los paladares realistas y se considera, además, una postura cercana al idealismo, o netamente idealista. ${ }^{26}$ En las secciones 5 y 6 de su texto, Sankey muestra qué caracterización prefiere del realismo y, al mismo tiempo, que consecuencias positivas (especialmente, en la lucha contra el escepticismo) tiene la adopción de esa perspectiva. Finalmente, se ocupa, en su última sección, del realismo directo, o natural, de la última fase de Putnam. Sobre esto último volveremos más adelante.

\section{LA PLURALIDAD DE ESQUEMAS CONCEPTUALES \\ Y EL A PRIORI CONTEXTUALIZADO}

En la fase del realismo interno, Putnam renuncia a dos de las tesis principales del realismo metafísico, tal como él lo define: (1) la existencia de una totalidad fija de objetos que (2) un único discurso es capaz de reflejar. Por otro lado, ofrece una revisión de la teoría de la verdad que abandona la clásica visión de la correspondencia (3) y abraza una semántica verificacionista. Con ello, inicia una transición hacia una comprensión de la pluralidad de discursos y del carácter relativo del contenido conceptual, y de las afirmaciones de la verdad, a ellos. Lo que decimos, el tipo de objetos y de propiedades de los que hablamos depende de un substrato conceptual común a los hablantes de un mismo lenguaje. Ese substrato conceptual es denominado con una vieja expresión muy a menudo utilizada: "esquema conceptual". Al adoptar un esquema conceptual nos comprometemos con la existencia de un cierto tipo de objetos.

Hay, en apariencia, dos consecuencias relacionadas entre sí que nacen de la adopción de la mecánica semántica y epistemológica basada en la noción de esquema conceptual: el abandono del realismo y el acercamiento al relativismo, tanto conceptual como epistémico. Pero Putnam, que no deja de ser un realista científico, no abandona esta perspectiva. Defenderá así, incluso muchos años después de la fase del realismo interno, que el realismo es compatible con esta perspectiva

${ }^{26}$ Ian Hacking (1983, p. 92) considera la postura de Putnam bajo esa denominación; Alan Musgrave, tal como muestra Sankey en su texto, también. 
y la clave es que el supuesto de que nuestras afirmaciones, si bien describen una cierta situación real en el vocabulario restringido de un esquema conceptual, nos remiten a esa situación real. Como Putnam decía: "Lo que significa ser un realista que reconoce la relatividad conceptual [...] es creer que hay un aspecto de la realidad que es independiente de lo que pensamos en cada momento [...], que se puede describir correctamente de cualquier manera". ${ }^{27}$

Hay, por lo tanto, una clara distancia entre el relativismo conceptual y lo que Putnam defiende, que es la relatividad conceptual, en la que no tiene cabida un concepto empleado por los defensores de la primera corriente, como Thomas Kuhn o Paul Feyerabend, que es la inconmensurabilidad. La razón para ello es que, como dice Ben-Menahem, "la idea clave de la relatividad conceptual de Putnam es diferente. Los diversos lenguajes autorizados no compiten realmente y se supone desde un principio que las descripciones que son formuladas en estos lenguajes son consistentes". ${ }^{28}$ Putnam, por lo tanto, intenta mostrar que nuestros modos de descripción de una misma situación adoptan diferentes discursos; que los intereses diversos que reflejan esos discursos hacen que nos sean más útiles unos que otros en distintos contextos humanos (las descripciones científicas, por exactas y verdaderas que puedan ser, no siempre aparecen como las idóneas en ciertos contextos); y que las dificultades, en ocasiones insuperables, que hallamos a la hora de intentar convertir uno de ellos en el lenguaje del otro (por ejemplo, la descripción en física teórica de un objeto de sentido común, como una silla) no deben obstaculizar el hecho de que existe esa misma situación, que es susceptible de ser reflejada, representada, de dos (o más) maneras diversas. ${ }^{29}$

En esta visión pluralista de los lenguajes y de su relación con el mundo cabe hacerse preguntas acerca de las transiciones de unos a otros, sobre todo en el caso del lenguaje de la ciencia. Esta misma pregunta condujo a los citados Kuhn

27 Putnam (2012a, p. 28).

28 Ben-Menahem (2015, p. 473). Sobre la diferencia entre el relativismo y la relatividad conceptual, cf. Button (2013, caps. 18-19) y Baghramian (2004, pp. 235-240).

${ }^{29}$ Cf. Putnam (2012a, pp. 28-29) y Ben-Menahem (2015, pp. 469-474). Baghramian (2004, pp. 237-239) que ha sido también una fuente, como las dos anteriores, para las ideas de este párrafo, cita un pasaje interesante de Realism with a Human Face, donde Putnam dice que: "Un corolario de mi relatividad conceptual [...] es la doctrina de que dos enunciados que son incompatibles en su sentido literal pueden a veces ser ambos verdaderos" (Putnam 1990b, p. x; cit. por Baghramian 2004, p. 237). Putnam establece distinciones entre la relatividad conceptual y el pluralismo conceptual, pero no entraremos en ellas aquí; a este respecto, $c f$. Putnam (2004, conf. 2; 2012a, pp. 29-30). 
y Feyerabend, sin ir más lejos, a proponer tesis tan radicales e incompatibles con el realismo científico como la ya mencionada inconmensurabilidad de las teorías científicas. No es el único ejemplo, por otro lado. Los cambios revolucionarios en la física de comienzos del siglo Xx condujeron a P. W. Bridgman o a los positivistas lógicos a replantearse la naturaleza de los enunciados que se consideran creencias fundamentales del pensamiento y la investigación científicas. Tanto en el caso de Kuhn como en el de varios positivistas lógicos (como Rudolf Carnap, Moritz Schlick o Hans Reichenbach), una de las preguntas centrales fue qué hacer con el concepto de juicio sintético a priori que había figurado como pieza clave del razonamiento de Immanuel Kant en la Crítica de la rąón pura.

Putnam, desde luego, no fue ajeno a esta última discusión. El artículo de Pablo Melogno, “A priori trascendental y a priori convencional. El intrincado camino de lo a priori de Kant a Putnam", se ocupa de esta interesante serie de reflexiones por parte de nuestro autor, que abarca un periodo considerablemente amplio.

La relación entre el a priori y el esquema conceptual, muestra Melogno, es estrecha en Putnam. Este se ocupa de mostrar cómo ciertas proposiciones adquieren un estatus de a priori debido al papel central que cumplen dentro de un cierto esquema conceptual: un papel de fundamentos, en ausencia de los cuales no tendría sentido hablar de ese preciso esquema conceptual. El a priori, en otras palabras, lo es por referencia (esto es, de forma relativa) a un determinado contexto. De ahí que cobre sentido hablar, en el caso de Putnam, de un a priori contextualizado, como deja claro Melogno.

Uno de los matices relevantes en esta concepción de Putnam es la idea de que aquellos enunciados, principios o creencias que consideramos "irrevisables" no lo son de hecho y que los cambios revolucionarios que se producen en la ciencia nos muestran que algunos de esos pilares fundamentales de una teoría (o de una visión del mundo) son sometidos a sustitución por otros más apropiados. Putnam propone como ejemplo cómo la lógica cuántica es capaz de poner de manifiesto que las leyes de la lógica booleana no tienen un estatus irrevocable; lo mismo ocurre con la geometría euclidiana en relación con las no euclidianas. Si hablamos del a priori en esos casos, hablamos de enunciados que no pueden ser abandonados a menos que aparezca una teoría alternativa idénticamente exitosa (o más). En tales casos, no podemos hablar de "irrevisabilidad" en términos absolutos; pero si podemos hablar de que los enunciados afectados eran "irrevisables" en términos relativos al contexto: eran, como dice Putnam, "contextualmente a priori". Así, "hay enunciados en la ciencia", explica Putnam, "que solo pueden ser derrocados por una nueva teoría [] y no solo por la observación. Tales enunciados tienen una clase 
de 'aprioridad' previa a la invención de la nueva teoría que los pone en duda o los sustituye: son contextualmente a prior". ${ }^{30}$

Melogno explica en su texto las particularidades del concepto de a priori que es visible en Putnam, una aclaración ya de por sí valiosa, pero además añade algo idénticamente significativo: su contraste con concepciones alternativas del a priori, desde su reemplazo por el punto de vista convencionalista que podemos apreciar en Henri Poincaré, en Schlick o en Carnap, hasta el plan de Reichenbach para un "a priori relativizado" en su Relativitätstheorie und Erkenntnis Apriori que, no obstante, fue abandonado después en pos de un avance hacia el convencionalismo. ${ }^{31}$ Melogno muestra que para Putnam, y no para Reichenbach, el a priori depende del esquema conceptual y, por lo tanto, no se enfrenta de manera directa a su potencial experiencia refutatoria. Esta dependencia en la perspectiva de Putnam del $a$ priori con respecto al esquema conceptual conduce a Melogno a defender, en sus conclusiones, una relación de mayor proximidad del filósofo estadounidense a Kant, en lo que se refiere a esta perspectiva sobre el a priori, que sus predecesores y mentores lógico-positivistas y lógico-empiristas.

\section{UNA "ILUSTRACIÓN PRAGMATISTA"}

En la obra de Putnam, las referencias a los autores clásicos del pragmatismo son abundantes, aunque también es cierto que suelen ser posteriores a 1978. Así, por ejemplo, en sus Philosophical Papers (volúmenes 1 y 2), es decir, en los escritos representativos de su periodo de mayor defensa del realismo metafísico (junto con el externismo en materia semántica), se puede observar una única mención a John Dewey (por nombrar a un representante de la corriente) y es para caracterizar la versión antirrealista de la verdad gracias a la "asertabilidad garantizada" de este. ${ }^{32}$ Ya en la segunda fase de Putnam, cuando la concepción de Dewey y la suya de "aceptabilidad racional" se aproximan como sustitutos de la verdad entendida al modo correspondencionalista, el primero ya no aparece como representante de

\footnotetext{
30 Putnam (1976b, p. 95; cit. por Melogno en su artículo para este número de Análisis). Para el punto de vista (y las expresiones) empleadas en este párrafo, cf. Putnam (1976b, pp. 91-95). 31 El libro de Reichenbach fue publicado por Springer en 1920 y traducido por Maria Reichenbach para la Universidad de California en 1965. La reconstrucción de este camino del $a$ priori al punto de vista convencionalista ha sido estudiado en profundidad por Michael Friedman, como también refleja Melogno; cf. Friedman (1999a, 1999b y 2001, esp. conf. II).

32 Cf. Putnam (1975d, p. 236). Véase también la mención a Dewey, con idéntico objetivo, al principio de Meaning and the Moral Sciences (Putnam 1978, p. 1).
} 
una postura por completo alejada de la del segundo. De ese modo, los pasajes de Reason, Truth and History y (en mayor medida) The Many Faces of Realism que mencionan a Dewey (en un par de ocasiones en cada caso) lo hacen ya como inspirador de aspectos de su propia postura. ${ }^{33}$ Con el salto a su tercera fase, Putnam acabará por defender lo oportuno de una corriente filosófica que seguiría a la filosofía platónica y a la Ilustración como un tercer movimiento ilustrado. Putnam denomina a este movimiento "tercera ilustración" o "ilustración pragmatista" y sitúa a Dewey como su principal representante. ${ }^{34}$

Si algo caracteriza a esta ilustración es su deseo de compartir algunos de los ideales reformistas del movimiento platónico y de la Ilustración, como por ejemplo su defensa encarnizada de una crítica racional de las ideas y de la organización democrática del estado, ${ }^{35}$ pero también la actitud contraria a algunos dogmas instalados en la filosofía que sirvió de base a, por ejemplo, su precedente en el siglo XVIII. En este último sentido, la ilustración pragmatista, de la mano de un representante como Dewey, aboga por una epistemología "falibilista y antiescéptica", 36 a diferencia de, por ejemplo, la perspectiva epistemológica empirista (que tanto este como Putnam critican). ${ }^{37}$ Además, el pragmatismo (su particular "ilustración”) se opone a la idea, idénticamente arraigada en el empirismo, de que hay (si usamos los términos de Putnam) un "interfaz" de sensaciones, o de datos sensoriales, "entre nuestras capacidades cognitivas y el mundo externo", por lo que esas "capacidades cognitivas no pueden acceder completamente a los objetos en sí mismos". 38 Serán Dewey y, antes que este, William James, los que critiquen esa concepción del "interfaz". 39 Putnam se suma a esta crítica y aboga por el realismo natural que surge de ella, principalmente de la mano de James, como veremos enseguida. ${ }^{40}$

\footnotetext{
33 Véase el texto de Faerna, a continuación en este número de Análisis (esp. sec. 1, n. 6), quien también se refiere a las pocas referencias a los autores pragmatistas en esta fase de Putnam.

${ }^{34}$ Putnam (2004, p. 96). La parte II de Ethics Without Ontology (Putnam 2004), esto es, sus Conferencias Spinoza de 2001, explican su visión de una “ilustración pragmatista” con detalle.

35 Cf. Putnam (2004, pp. 94 y 97).

36 Putnam (2004, p. 99); cursiva en el original.

37 Así, de modo semejante a Dewey, Putnam es crítico hacia la consideración de distinciones como hecho/valor (o, en el caso de este último, analítico/sintético) como "dicotomías metafísicas”. Cf. Putnam (2002a, pp. 9-13 y esp. p. 11 para la expresión entrecomillada).

38 Putnam (1999, p. 10; 2001, p. 12).

39 Cf. Putnam (2004, pp. 98-99).

${ }^{40}$ Cf. Putnam (1999, conf. 1, esp. p. 10).
} 
Esta última concepción realista muestra que Putnam no ha abandonado su inicial convicción de que debemos mantener un compromiso con la existencia de un mundo externo con entidades que logramos describir mediante los esquemas conceptuales adecuados. Como muestra en sus argumentos acerca de la relatividad conceptual, quizá no tenga sentido formular preguntas relativas a qué esquema conceptual refleja mejor cómo son las cosas en si mismas, preguntas, estas, que carecen de sentido para él desde su etapa del realismo interno; sin embargo, continúa teniendo sentido afirmar que, una vez que un esquema conceptual constituye nuestro fundamento en materia ontológica, la pregunta de, por ejemplo, qué objetos existen en el mundo es una cuya respuesta puede quedar perfectamente definida, al margen de lo que pudiéramos decidir que queremos responder. En otras palabras, en el seno de un esquema conceptual, qué existe y qué no es ya una materia de decisión consensuada y, por lo tanto, no se ofrece a las libertades del relativista. El uso de "existe" (por usar uno de los modos equivalentes de aportar una lectura del cuantificador existencial en un lenguaje cotidiano) queda así, como apunta nuestro autor, fjjado en uno de sus múltiples sentidos. ${ }^{41}$ Una vez dentro de ese marco, podemos seguir una indicación ya visible en la última obra de Carnap y en la última obra de Kuhn: que las preguntas sobre el marco en sí, es decir, sobre el propio esquema conceptual y su capacidad para reflejar cómo son las cosas en el mundo al margen suyo, carecen de sentido, mientras que las preguntas internas al marco (esto es, qué existe, por ejemplo) se responden de acuerdo con criterios de verdad inequívocos. ${ }^{42}$

Es importante subrayar una diferencia clave de este periodo del realismo natural con respecto al del realismo interno: en este caso, los esquemas conceptuales no actúan como una "interfaz" más del tipo arriba referido; más en concreto, no actúan como parte de una "interfaz" en la que se integran por igual las experiencias y los conceptos, según el matiz de Putnam. Los esquemas conceptuales nos permiten tener acceso selectivo al mundo, pero no como intermediarios, sino como formas de adentrarnos en el mundo. Como Putnam muestra en "James's Theory of Perception" (1990), James ya mostró que no había fundamento convincente para sostener la idea de dicho intermediario, aunque fuese parte de la tradición filosófica arraigada en el empirismo y que llega hasta comienzos del siglo xx..$^{43}$ Así, Putnam confiesa que, en un primer momento, él tampoco logró ver que (en sus palabras),

41 Sobre los usos de "existe", $c f$. Putnam (2004, pp. 37 y ss.).

42 Para las ideas resumidas en este párrafo, $c f$. Putnam (1987, pp. 17-20; 2004, parte I, conf. 2).

43 Cf. Putnam (1990a, esp. pp. 242-246) 
aunque "las experiencias están conceptualizadas", no obstante se "puede pensar en las experiencias y los conceptos como formas - por usar lo que creo que es el lenguaje de [John] McDowell— de apertura al mundo". "44 Más adelante, pudo darse cuenta de lo acertado de esa visión. ${ }^{45}$

En esta perspectiva, por lo tanto, se repite una tesis que Putnam ha expuesto desde comienzos de los años 80: la defensa de la pluralidad de esquemas conceptuales y de la relatividad conceptual no implica una defensa del relativismo conceptual. Eso lo sitúa en una posición contraria a la de Richard Rorty, aunque ambos defiendan la perspectiva heredada del pragmatismo. ${ }^{46}$ A este respecto, Putnam es bastante explícito: "Aunque normalmente no me llamo a mí mismo pragmatista [], no me importa cuando me describen así, excepto si la gente asimila mis perspectivas a las de [...] Richard Rorty". ${ }^{47}$ Precisamente, el capítulo de Ángel Faerna, "Hilary Putnam y la nostalgia del pragmatismo", refleja la (voluntaria) lejanía de Putnam con respecto a Rorty en el seno de la corriente pragmatista.

En el artículo de Faerna vemos que Putnam deseó cada vez más situarse al margen de una perspectiva puramente analítica y que el pragmatismo, una corriente que no lo sedujo en un principio demasiado, fue un buen aliado en esa transición. Faerna muestra que podemos entender el obstáculo del Putnam de las primeras décadas para aproximarse al pragmatismo: como consumado lógico, como filósofo reconvertido en matemático con más que notables resultados y como seguidor inicial del positivismo lógico (convencido por la versión que ofreció de este A. J. Ayer y con Hans Reichenbach como director de tesis, recuerda Putnam) y, más tarde, del realismo entendido al modo analítico, no es sorprendente que se hallase en tierra extraña al leer por primera vez la Logic de Dewey y que le costase, como también confiesa, ver que los hechos sí estaban enredados con los valores. ${ }^{48}$ Faerna explora a fondo estos datos biográficos desde el punto de vista de las relaciones entre la tradición analítica en suelo estadounidense y su autóctona tradición pragmatista para explicar la evolución de Putnam hacia algunas tesis de esta última corriente.

${ }^{44}$ Putnam (2012a, p. 27). Para la expresión de McDowell, cf., por ejemplo, McDowell (1996, p. 111).

${ }^{4}$ Cf. Putnam (2012a, pp. 26-27), sobre lo dicho en este párrafo.

46 Aunque, de acuerdo con el propio Putnam, el reflejo del pragmatismo es menos preciso en el caso de Rorty. Cf. Putnam (2012a, p. 33).

47 Putnam (2012a, p. 33).

48 Para este relato de sus primeros (y tímidos) pasos en el pragmatismo, cf. Putnam (2002b, p.

13) y el comienzo del texto de Faerna para este número de Análisis. 
Uno de los aspectos discutidos en el artículo de Faerna tiene que ver con el modo en que Putnam cree que el pragmatismo puede contribuir a los temas clásicos de la epistemología más que suprimir esta última como un proyecto ya caduco, una postura, esta última, en la línea de Rorty. Ya hemos observado que el vínculo de la concepción de la verdad en el Putnam del periodo del realismo interno implica una aproximación a las tesis de Dewey sobre la asertabilidad garantizada. Para Rorty, este último concepto de Dewey supone un guiño a una concepción de la filosofía en la que el concepto de verdad deja de cumplir un papel central. No es así para Putnam, cuyos argumentos en Reason, Truth and History dejan ver que un concepto semejante, la aceptabilidad racional, puede llenar el hueco que deja tras de sí la teoría de la verdad como correspondencia con el abandono del realismo metafísico. Ahora bien, Faerna deja claro que ninguno de ambos, ni Rorty ni el propio Putnam, consiguen hacer justicia por completo a Dewey y, sobre todo, a la dependencia de la asertabilidad garantizada con respecto a la teoría de la verdad de Peirce. Faerna muestra la íntima dependencia de la primera con respecto a la segunda en la medida en que Dewey incide en (i.e., investiga a fondo) los rasgos normativos que permiten hablar de "comunidad" en el pensamiento de Peirce, esto es, un grupo humano que persigue el desarrollo de una creencia que no genere dudas y que es producto de métodos de investigación no rígidos, sino elaborados poco a poco, habida cuenta de su carácter falible. La dimensión "ética" (en sentido epistemológico) que integra a los miembros individuales de la "comunidad" en el sentido de Peirce no reconduce a los estándares de justificación de la creencia a un enfoque relativista, sino a la persecución de un ideal de investigación y de una versión siempre mejorada del método científico. Esta importante idea central en la obra de estos dos pragmatistas clásicos no fue, así pues, bien entendida por Rorty y tampoco por Putnam.

Los rasgos del enfoque que caracteriza la "tercera ilustración" se ponen de manifiesto en los vínculos que Putnam establece con autores como James o Dewey. Su perspectiva, aunque en 1990 ya se aleje del realismo interno, permite que perviva las ideas de un pluralismo de esquemas conceptuales y de la relatividad conceptual. Es cierto, como varias veces escribe, que ahora aborrece la expresión metafórica "la mente y el mundo construyen conjuntamente la mente y el mundo". ${ }^{49}$ Sin embargo, eso no implica que se declare a favor de un realismo metafísico como el que criticó a partir de 1976. Su realismo, como ya hemos

49 Putnam (1981, p. xi; 1988, p. 13); sobre el abandono de dicha afirmación, cf. Putnam (2012a, pp. 25-29). 
advertido, ha evolucionado hacia el tipo de compromisos reducidos con los que cuenta, por ejemplo, Sankey y, lo que es más importante, a la supresión de un intermediario para nuestra relación con el mundo que hace del carácter "directo" de dicha relación (perceptiva) con los objetos una postura más que plausible. La relación con el mundo no desaparece de su perspectiva, ni tampoco lo hace la idea de que dicho mundo puede ser descrito mediante diversos sistemas de conceptos (o esquemas conceptuales), algunos de los cuales pueden, simplemente, ser incompatibles o, en general, hallar pocos vínculos entre sí, al margen, claro está, de referirse a un mundo común a todos.

La postura que conlleva esta idea es la que expone en Ethics Without Ontology como "pluralismo pragmático" y que constituye una alternativa al recurso al pensamiento metafísico que caracterizó, por ejemplo, la primera de las corrientes ilustradas que precedieron a la pragmatista: la metafísica platónica. La perspectiva del pluralismo pragmático rehúye la explicación filosófica basada en recursos de naturaleza ontológica y abraza una pluralidad de discursos en las que se cifran nuestros modos de hablar de cuestiones científicas o de aspectos del sentido común. De la ciencia, en sus diversos campos (e incluida en ello la matemática) a la ética, no podemos contar más que con un ámbito variado de formas de discurso que no se someten con facilidad a teorías filosóficas basadas en ontologías unificadas. Putnam aboga, de este modo, una vez más, por la relatividad conceptual y por el pluralismo. ${ }^{50}$

El artículo de David Macarthur, "The Many Faces of Objectivity", se ocupa precisamente de este pluralismo pragmático, con especial atención a nuestras prácticas normativas. Macarthur muestra cómo Putnam defiende que no hay una única teoría, ya sea basada en determinados compromisos ontológicos, ya sea asentada en cierto tipo unificado de reglas del método, que sea capaz de dar cuenta, de forma general, de los modos en que podemos responder a las preguntas sobre la naturaleza y vínculo entre conceptos como los de "verdad” y "justificación”. Macarthur explora en su texto los diferentes estadios de la obra de Putnam en los que ha explicado la relación entre esos dos conceptos y su relación con las formas del realismo defendidas en cada momento. Para Macarthur, Putnam muestra (sobre todo en su segunda fase) el modo de rechazar el realismo metafísico sin que ello desemboque en una postura relativista. La tarea que encara Putnam en adelante es la de hallar un camino intermedio que muestre cómo puede ser explicada la normatividad en ese escenario plural sin incurrir en uno de esos dos extremos (el

50 Sobre esta postura, of. Putnam (2004, pp. 21-22 y, para mayor detalle, pp. 15-22). 
realismo metafísico y el relativismo). Macarthur es, no obstante, crítico con Putnam, debido a que defiende que este no abandona por completo el debate entre realismo y antirrealismo e intenta construir una alternativa que ofrezca respuestas sustantivas a la pregunta acerca de las relaciones del lenguaje (y la mente) con el mundo, a pesar de que la idea de una metafísica inflacionista ya no esté, en efecto, presente.

\section{PUTNAM, EL REALISMO CIENTÍfICO Y LA TEORÍA CUÁNTICA}

Hasta ahora, hemos visto principalmente cómo desarrolla Putnam su visión del realismo (y sus consecuencias) y cómo, en ese proceso, la perspectiva pragmatista es una incorporación que, aunque tardía (al menos con respecto a algunos de sus recursos y conocimientos más representativos, como la lógica, las matemáticas o la tradición analítica), ha dejado una huella profunda en su pensamiento filosófico. Algunos de los ensayos que siguen a este artículo de introducción se centran en esa exploración putnamiana del realismo y del pragmatismo (y de sus implicaciones). Otros dos, en cambio, de los que hablaremos en esta última sección, se ocupan de debatir el trabajo de Putnam en un terreno al que aplicó sus conocimientos y su perspectiva filosófica (y, de manera predominante, la realista). Nos referimos a la teoría cuántica. Este es el tercer aspecto de la obra de Putnam al que algunos de los ensayos de este número de Análisis dedican su atención.

En efecto, uno de los campos de investigación, en este caso en filosofía de la ciencia, a los que Putnam ha dedicado considerable esfuerzo ha sido la teoría cuántica. En especial, Putnam ha tratado en varias ocasiones las posibilidades de una interpretación realista de la mecánica cuántica. En sus palabras: "Desde 1960, [] el área de la filosofía de la física que me ha interesado más es la mecánica cuántica y el problema de extender a esa materia el realismo científico es uno en el que he pensado y seguido discutiendo con amigos desde entonces". ${ }^{11}$ Putnam subraya que su enfoque es el de un realista científico en varias ocasiones ya solo en su biografía intelectual (cf. Putnam 2015a, pp. 65-67).

Putnam ha dedicado un buen número de textos a la teoría cuántica, algunos de los cuales pueden encontrarse en los volúmenes 1 y 3 de sus Philosophical Papers (en especial, en el primero de ambos), pero son bien conocidos, en particular, dos de ellos, íntimamente relacionados (y no solo debido al título), entre los que median cuatro décadas exactas: "A Philosopher Looks at Quantum Mechanics"

51 Putnam (2015a, p. 65). 
(1965) y "A Philosopher Looks at Quantum Mechanics (Again)" (2005). En ellos, Putnam muestra una posición realista que tiene como principal caracterización, como él dice, "el rechazo del operacionalismo". ${ }^{52} \mathrm{El}$ segundo artículo siguió al primero porque, en su opinión, en esos cuarenta años le habían ocurrido demasiadas cosas a la teoría que era preciso tener en cuenta. ${ }^{53}$ La contribución de Javier García Sanz a este número de Análisis, "Algunas notas sobre Putnam y la mecánica cuántica", se dedica, precisamente, a actualizar algunas de las conclusiones de Putnam acerca de problemas como el de la medida o sobre el uso de algoritmos cuánticos. El texto de García Sanz pone al día las previsiones de Putnam en esta materia, al igual que algunos aspectos de sus interpretaciones.

El texto de García Sanz es uno de los dos artículos dedicados al trabajo de Putnam con la filosofía de la mecánica cuántica. El otro ensayo es el de Richard Healey y se ocupa del punto de vista con el que comenzábamos esta sección: la perspectiva realista que Putnam adopta a la hora de discutir dicha teoría. El artículo de Healey se titula "Quantum Theory: Realism or Pragmatism?” y su objetivo, como refleja su título, es mostrar lo oportuno de una interpretación pragmatista de la mecánica cuántica. En efecto, en un pasaje de su "Quantum Mechanics and Ontology", Putnam observaba dos alternativas para la interpretación de la función de onda; de acuerdo con una de ellas, "a la función de onda se le da una 'interpretación empírica' mediante 'definiciones coordinadoras', tal como Carnap habría dicho". A continuación decía Putnam: "Pero esta no es la clase de explicación que un realista científico —alguien que quiere comprender la mecánica cuántica como algo que describe la realidad y no solo como un recurso para hacer prediccionesestá buscando. Y yo soy un realista científico". ${ }^{54}$ En su artículo, Healey defiende la posibilidad de una tercera vía además de las dos posturas así ofrecidas: las del realismo putnamiano y las de un instrumentalismo al modo de Carnap.

La perspectiva de Healey tiene que ver con la concepción realista de algo más general: el propio desarrollo de la ciencia. Healey se hace eco, al principio de su ensayo, de la perspectiva de Putnam, en conjunción con la de Richard Boyd,${ }^{55} \mathrm{del}$

\footnotetext{
52 Putnam (2015a, p. 67). En el más reciente de los dos (Putnam 2005), Putnam cita un largo párrafo en la segunda sección del más antiguo de ambos artículos (Putnam 1965; cf. Putnam 2005, pp. 128-130) en el que recupera esa postura realista con ese preciso sentido.

53 Putnam (2005, p. 126; 2015a, p. 67).

54 Putnam (2012b, p. 151). Healey reproduce, en la segunda sección de su artículo, este pasaje de Putnam de una manera más completa.

55 Sobre la propuesta de Boyd y su relación con la de Putnam, cf. Psillos (1999, cap. 4).
} 
realismo científico como una visión del desarrollo científico que explica bien el éxito de la ciencia y que muestra que vamos, poco a poco, acotando mejor la ontología que ha de aparecer en nuestra visión científica del mundo. Healey introduce una variación que califica como pragmatista. Para él, algunas de nuestras mejoras en esa visión científica —y la mecánica cuántica es, sin ningún género de duda, una de ellas - no se entienden de la mejor manera posible desde el punto de vista de una aportación de nuevos elementos a dicha ontología (lo que sería una comprensión del progreso al modo realista), sino desde el punto de vista de una mejora de los recursos teóricos ya existentes. Healey muestra que esa interpretación posee credenciales pragmatistas y que constituye una posibilidad que discurre entre las dos indicadas por Putnam en pasajes como en el que hemos leído en el párrafo anterior.

\section{CONSIDERACIONES FINALES}

Como dijimos en un principio, este ensayo constituye una introducción a algunos de los temas que Putnam trató a lo largo de su carrera. Normalmente, como es visible a lo largo de esta presentación y, más clara y detalladamente, en los seis ensayos que la siguen, Putnam volvió una y otra vez sobre los mismos problemas o enfoques y, en más de una ocasión, con un enfoque renovado que añadía nuevas materias de discusión. Como en el momento de la aparición del pragmatismo (o, para ser más precisos, de algunos modelos de tratamiento de los problemas de la filosofía propios de autores adscritos al pragmatismo) en su obra, nuevos comentaristas y críticos se sumaron a la discusión de sus propuestas. Dada la amplia gama de cuestiones debatidas por Putnam, de la epistemología a la ética y de la lógica a la teoría cuántica, un tratamiento apropiado, aunque se limite a unas pocas cuestiones, como el que ofrecemos en este número de Análisis, requiere la colaboración de un buen número de especialistas.

Por eso, desde estas páginas deseamos agradecer a los colaboradores de este número su buen hacer y su amable disposición. Desde un primero momento, Ángel Faerna, Javier García Sanz, Richard Healey, David Macarthur, Pablo Melogno y Howard Sankey mostraron su deseo de ofrecer ensayos sobre la figura y la obra de Hilary Putnam en sus áreas de especialización. A todos ellos, gracias. No obstante, desearíamos hacer una mención especial a Javier García Sanz, hoy lamentablemente fallecido. Javier entregó su ensayo para este número en la primavera de 2017, pero falleció el verano siguiente. Su magnífica contribución habla por sí sola, aunque no es solo su brillantez e inteligencia lo que echaremos de menos, 
sino también su amabilidad y humanidad. Nos gustaría dedicar este número de homenaje a Putnam a la memoria de Javier. ${ }^{56}$

\author{
Juan V. Mayoral \\ Universidad de Zaragoza \\ jmayoral@unizar.es
}

\title{
BIBLIOGRAFÍA
}

Auxier, R. E., D. R. Anderson y L. E. Hahn (eds.) (2015): The Philosophy of Hilary Putnam, The Library of Living Philosophers, Volumen XXXIV, Chicago, IL: Open Court.

Baghramian, M. (2004): Relativism, Londres: Routledge.

Baghramian, M. (2013a): "Introduction: a Life in Philosophy", en Baghramian (2013b, pp. 1-16).

Baghramian, M. (2013b): Reading Putnam, M. Baghramian (ed.), Londres: Routledge.

Baghramian, M. (2016): "Hilary Putnam (1926-2016)", Philosophy Now, Junio-Julio, pp. 48-49.

Ben-Menahem, Y. (2015): “Revisiting the Refutation of Conventionalism”, en Auxier, Anderson y Hahn (eds.) (2015, cap. 12 [pp. 451-478]).

ButTon, T. (2013): The Limits of Realism, Oxford: Oxford University Press.

De CAro, M. (2016): “Introduction: Putnam's Philosophy and Metaphilosophy", en Putnam (2016b pp. 1-18).

De Caro, M., y D. Macarthur (2012): "Introduction: Hilary Putnam: Artisanal Polymath of Philosophy”, en Putnam (2012b, pp. 1-35).

Devit'T, M. (1991): Realism and Truth, $2^{\mathrm{a}}$ ed., Princeton, NJ: Princeton University Press.

DiÉGuEZ, A. (1998): Realismo científico. Una introducción al debate actual en la filosofía de la ciencia, Málaga: Servicio de Publicaciones de la Universidad de Málaga.

Friedman, M. (1999a): “Geometry, Convention and the Relativized A Priori: Reichenbach, Schlick, and Carnap”, en Friedman (1999c, cap. 3 [pp. 59-70]).

FrIEDman, M. (1999b): "Poincaré's Conventionalism and the Logical Positivists", en Friedman (1999c, cap. 4 [pp. 71-86].)

FrIEDman, M. (1999c): Reconsidering Logical Positivism, Cambridge: Cambridge University Press.

Friedman, M. (2001): Dynamics of Reason. The 1999 Kant Lectures at Stanford University, Stanford, Cal.: CSLI Publications.

${ }^{56}$ Este trabajo se ha realizado en el marco del proyecto "Ciencia, cultura y valores (II): Un enfoque pragmatista sobre la normatividad científica” (FFI2017-84781-P), financiado por el Ministerio de Economía, Industria y Competitividad. 
HACKING, I. (1983): Representing and Intervening, Cambridge: Cambridge University Press. HACKInG, I. (1996): Representar e intervenir, trad. Sergio Martínez de Hacking (1983), México: Paidós/UNAM.

HORWICH, P. (1990): Truth, $2^{\mathrm{a}}$ ed., Oxford: Oxford University Press.

MCDoweld, J. (1996): Mind and World, $2^{a}$ ed., Cambridge, Mass.: Harvard University Press. Psillos, S. (1999): Scientific Realism: How Science Tracks Truth, Londres: Routledge.

Putnam, H. (1960): “What Theories Are Not”, en Putnam (1975c, cap. 13 [pp. 215-227]). Putnam, H. (1965): "A Philosopher Looks at Quantum Mechanics", en Putnam (1975c, cap. 7 [pp. 130-158]).

Putnam, H. (1975a): “What Is Mathematical Truth?”, en Putnam (1975c, cap. 4 [pp. 60-78]).

Putnam, H. (1975b): “Introduction: Science as Approximation to Truth", en Putnam (1975c, pp. vii-xiv).

Putnam, H. (1975c): Mathematics, Matter and Method: Philosophical Papers, Volume 1, Cambridge: Cambridge University Press.

Putnam, H. (1975d): “The Meaning of 'Meaning”, en Putnam (1975e, cap. 12 [pp. 215271]).

Putnam, H. (1975e): Mind, Language and Reality: Philosophical Papers, Volume 2, Cambridge: Cambridge University Press.

Putnam, H. (1976a): “Realism and Reason”, en Putnam (1978, Parte 2 [pp. 123-138]).

Putnam, H. (1976b): “'Two Dogmas’ Revisited”, en Putnam (1983, cap. 5 [pp. 87-97]).

Putnam, H. (1978): Meaning and the Moral Sciences, Cambridge, Cambridge University Press. Putnam, H. (1981): Reason, Truth and History, Cambridge: Cambridge University Press.

Putnam, H. (1982): “A Defense of Internal Realism”, en Putnam (1990b, cap. 2 [pp. 30-42]).

Putnam, H. (1983): Realism and Reason: Philosophical Papers, Volume 3, Cambridge: Cambridge University Press.

Putnam, H. (1988): Razón, verdad e historia, trad. J. M. Esteban de Putnam (1981), Madrid: Tecnos.

Putnam, H. (1990a): “James's Theory of Perception”, en Putnam (1990b, cap. 17 [pp. 232-251]).

PutNam, H. (1990b): Realism with a Human Face, ed. e introd., J. Conant, Cambridge, Mass.: Harvard University Press.

Putnam, H. (1991): El significado y las ciencias morales, trad. A. I. Stellino de Putnam (1978), México: UNAM.

Putnam, H. (1994): “Comments and Replies”, en Reading Putnam, P. Clark y R. Hale (eds.), Oxford: Blackwell, cap. 10 (pp. 242-295).

Putnam, H. (1997): “A Half Century of Philosophy, Viewed from Within”, Daedalus, 126; cit. por la versión en castellano: 50 años de filosofía vistos desde dentro, trad. C. Castells, Barcelona: Paidós, 2001.

Putnam, H. (1999): The Threefold Cord: Mind, Body, and World, Nueva York: Columbia University Press. 
PuTNAM, H. (2001): La trenqa de tres cabos: La mente, el cuerpo y el mundo, Pról. de J. F. Álvarez y C. Gómez, trad. J. F. Álvarez de Putnam (1999), Madrid: Siglo XXI.

Putnam, H. (2002a): The Collapse of the Fact/V alue Dichotomy and Other Essays, Cambridge, Mass.: Harvard University Press.

Putnam, H. (2002b): “Comment on Ruth Anna Putnam's Paper”, en Hilary Putnam: Pragmatism and Realism, J. Conant y U. M. Zeglen (eds.), Londres: Routledge, pp. 12-13 (v.c.). Reeditado en Putnam y Putnam (2017, pp. 18-20).

Putnam, H. (2004): Ethics Without Ontology, Cambridge, Mass.: Harvard University Press.

Putnam, H. (2005): “A Philosopher Looks at Quantum Mechanics (Again)”, en Putnam (2012c, cap. 6 [pp. 126-147]).

PutNAM, H. (2012a): "From Quantum Mechanics to Ethics and Back Again", en Baghramian (2013, pp. 19-36; v.c.). Reeditado en Putnam (2012c, cap. 2 [pp. 51-71]).

Putnam, H. (2012b): "Quantum Mechanics and Ontology", en Putnam (2012c, cap. [pp. 148-161]).

Putnam, H. (2012c): Philosophy in an Age of Science: Physics, Mathematics, and Skepticism, Mario de Caro y David Macarthur (eds)., Cambridge, Mass.: Harvard University Press.

Putnam, H. (2013a): “The Development of Externalist Semantics”, en Putnam (2016, cap. 12 [pp. 199-212]).

Putnam, H. (2013b): "Sixty-Five Years of Philosophy: A Participant's Thoughts and References”, en Putnam (2016, cap. 13 [pp. 213-226]).

Putnam, H. (2015a): “Intellectual Autobiography”, en Auxier, Anderson y Hahn (eds.) (2015, parte I [pp. 3-110]).

Putnam, H. (2015b): “Reply to Yemima Ben-Menahem”, en Auxier, Anderson y Hahn (eds.) (2015, pp. 479-485).

Putnam, H. (2016a): “The Development of Externalist Semantics”, en Putnam (2016b, cap. 12 [pp. 199-212]).

Putnam, H. (2016b): Naturalism, Realism, and Normativity, M. De Caro (ed.), Cambridge, Mass.: Harvard University Press

Putnam, H., y R. A. Putnam, Pragmatism as a Way of Life: The Lasting Legacy of William James and John Dewey, David Macarthur (ed.), Cambridge, Mass.: Harvard University Press.

SANKEY, H. (2008a): “Scientific Realism”, en Sankey (2008d, cap. 1 [pp. 11-29]).

SANKEY, H. (2008b): "The God's Eye Point of View”, en Sankey (2008d, cap. 2 [pp. 31-41]).

SANKEY, H. (2008c): "Incommensurability and the Language of Science", en Sankey (2008d, cap. 4 [pp. 53-77]).

SANKEY, H. (2008d): Scientific Realism and the Rationality of Science, Aldershot: Ashgate.

Wright, C. (2000): “Truth as Sort of Epistemic: Putnam's Peregrinations", Journal of Philosophy, 97, pp. 335-364. 